\title{
Proyecto "GIRAgua”: síntesis de la cooperación internacional
}

\author{
Pablo Carrasco*
}

\section{Resumen}

El actual uso del agua, además de ser regido por una ley que no se adapta a las necesidades ni el contexto de abundancia de agua del país, como el Código de Aguas de 1982, genera presión adicional sobre las cuencas y acuíferos que son usados intensamente o sobreexplotados por la industria minera y la agroindustria; esto hace que sigan pensando en la disponibilidad del agua usando soluciones tecnológicas convencionales anticuadas, que no están acorde con la situación real de escasez del agua en Chile. Ante esta situación, el objetivo de este artículo es presentar al proyecto "GIRAgua" como un proyecto de cooperación internacional pactado entre el Ministerio de Obras Públicas chileno y el Ministerio de Infraestructura y Medio Ambiente neerlandés, mediante la denominada "Carta de Intenciones", firmada en julio del año 2016 como forma de promover el uso racional del agua y los conceptos de Gestión Integrada de los Recursos Hídricos y de Gobernanza del Agua; paradigmas claves para enfrentar el actual contexto de sobrecarga de acuíferos, uso intensivo del agua y suscitar la participación de la ciudadanía en el diseño de políticas públicas hídricas.

Palabras claves: Cooperación internacional; Proyecto GIRAgua; Gestión integrada de Recursos Hídricos; Gobernanza del Agua.

\begin{abstract}
The current use of water, in addition to being governed by a law that does not adapt to the needs or context of water abundance in the country, such as the 1982 Water Code, generates additional pressure on the basins and aquifers that are used intensively or overexploited by the mining industry and agribusiness; this keeps them thinking about the availability of water using outdated conventional technological solutions, which are not in line with the real situation of water scarcity in Chile. Considering this situation, the objective of this article is to present the "GIRAgua" project as an international cooperation project agreed between the Chilean Ministry of Public Works and the Dutch Ministry of Infrastructure and Environment, through the so-called "Letter of Intentions", signed in July 2016, as a way to promote the rational use of water and the concepts of Integrated Water Resources Management and Water Governance; key paradigms to face the current context of aquifer overload, intensive use of water and inspire the contribution of citizens in the design of public water policies.
\end{abstract}

Keywords: International cooperation; "GIRAgua” Project; Integrated Management of Water Resources; Water Governance.

\footnotetext{
* Master in International Studies, Institute of International Studies, University of Chile. Email: pablo.carrasco@ug.uchile.cl. Received: April, 14th 2019; modifications: May, 3 ${ }^{\text {rd }} 2019$; accepted: May, 6th 2019.
} 
Pablo Carrasco

Proyecto "GIRAgua": síntesis de la cooperación internacional

\section{Introducción}

La actual demanda sobre el agua en Chile no es homogénea. Mientras que desde la Región Metropolitana al norte del país prevalecen las "mega sequías" y disminuye el caudal de los ríos, la situación es diferente en el sur donde la disponibilidad "de O’Higgins hacia el sur superan los $7.000 \mathrm{~m}^{3} /$ persona/año, se llega a un valor de $2.950 .168 \mathrm{~m}^{3}$ / persona/año en la Región de Aysén”" (Ministerio de Obras Públicas, 2014, p. 8).

Además de contar con el problema de la "mega sequía", existen otros desafíos a nivel institucional: la actual legislación que se encarga de gestionar el acceso y distribución del agua conocido como el Código de Aguas, que fue creado en un contexto de "abundancia", sigue estando vigente aún en un contexto diametralmente diferente como es es el de crisis hídrica. La institucionalidad más importante del rubro la Dirección General de Aguas (DGA) - no tiene suficiente capacidad de fiscalización y monitoreo acerca de los derechos de agua previamente adquiridos por sus actuales propietarios, adolece de información precisa sobre los datos de cuencas, ríos y deltas, y debe encarar el mayor desafío: evitar una crisis mayor sobre la gestión del agua como recurso ya no económico, sino que social.

Ante ello y como respuesta a este desafío a nivel país, el año 2016, la Intendencia de la Región de Coquimbo pidió la evaluación a un panel de expertos holandeses para monitorear y evaluar los desafíos del problema del agua de forma integral, avanzando en el análisis "de la gestión de riesgos para inundaciones e inundaciones repentinas en el norte de Chile, con el apoyo de mecanismos como DRR (Dutch Reduction Risk Team), entre otros" (Ministerio de Obras Públicas de Chile, 2016, p. 1). La idea era generar un centro especializado chileno-neerlandés para el agua - Instituto Tecnológico Aplicado del Agua (ITA) - que se espera sea replicado en cada una de las regiones del país desde el año 2018 hasta el 2030. El ante proyecto técnico aprobado genera expectativas positivas entre ambas partes.

Pero, ¿por qué fijarse en los Países Bajos? ¿Qué hace que éste país sea un referente en el manejo integrado de los recursos hídricos? El experto en aguas neerlandés Henrik Ovink, advirtió por ejemplo: “(...) podríamos decir que la situación del agua es crítica, y al mismo tiempo no lo enfrentamos, no lo entendemos, hay una falta de conciencia, hay falta de gobernanza y manejo del agua, hay falta de conocimiento, información y visión" (Fajardo \& De Britto, 2017). Además, el experto menciona el caso chileno como una forma de destacar las carencias de expertise para afrontar la grave escasez hídrica por un parte, y abordar las consecuencias del cambio climático y la alta demanda de agua en la economía nacional.

Con respecto a los Países Bajos como pionero internacional en esta materia, Sybe Schaap, presidente del programa de cooperación internacional holandés "Netherlands Water Partnership" declara: "Nos aseguramos de que los intereses estén conectados, de que se logre la cooperación correcta. Nuestro lema es 'más impacto en el exterior'. El impacto es más que solo trabajo, más que solo un mercado, se trata de exportación y de que esto contribuye a soluciones sostenibles” (Schaap, 2016, p. 3). De esta manera sostiene que si solo actuaran los empresarios desde un ámbito puramente comercial, la gestión integrada del agua "no tiene respaldo, sino un enfoque puramente público que no cuenta con la tecnología y los inversores adecuados" (Schaap, 2016, p. 3). 
El concepto de Gestión Integrada de Recursos Hídricos -en adelante GIRH- se define como "un proceso que promueve el desarrollo y la gestión coordinados del agua, la tierra y los recursos relacionados, para maximizar el bienestar económico y social resultante de una manera equitativa sin comprometer la sostenibilidad de los ecosistemas vitales" (Mitchell, 2005, 9. 1336). En sus comienzos, el concepto de GIRH tuvo dos énfasis:

1. El primero, una aproximación de análisis de datos a través de informes técnicos para "proporcionar una base científica para una gestión óptima de cantidad y calidad de aguas superficiales y subterráneas"

2. En una segunda aproximación, la preocupación sobre la relación de la tierra, los individuos y la ecología tomó cada vez más importancia ya que "el término fue utilizado en relación con cuestiones como los sedimentos contaminados, la gestión ecológica activa y los bancos favorables a la naturaleza" (Mostert, 2006, p. 21).

Para constituir la GIRH como concepto fundamental en los Países Bajos, se requirió de una estrecha cooperación de todas las autoridades con competencia o influencia en el sistema de agua, una legislación y sistemas de financiación bien desarrollados. De esta forma "la protección contra inundaciones ha tenido éxito durante 60 años gracias a los cambios en la organización, la legislación, los instrumentos financieros y los institutos. La gestión de los riesgos de inundación y la gestión del agua están integradas en nuestro país" (Slomp, 2012, p. 3).

Además, como concepto central de la Política Hídrica de los Países Bajos, la GIRH "se considera y se presenta como una ruptura con el paradigma tecnocrático, orientado a la oferta y orientado a la construcción del pasado, para señalar una nueva era en la que las preocupaciones económicas, sociales, ambientales y sociales se abordan simultáneamente y en sus interacciones mutuas" (Zwarteveenn \& Boelens, 2014, p. 145). Mas este concepto no es una prospectiva, sino que un camino a seguir, ya que "dada la actual crisis del agua, la experiencia del agua holandesa es más necesaria que nunca. Juntos aseguramos un mayor impacto en el exterior" (Schaap, 2016, p. 3). Para desarrollar este concepto en un país como Chile, es necesario saber cómo ejecutar un plan de este tipo. Para la implementación o ejecución de esta política pública hay dos pasos:

1. Identificar metas o direcciones; $y$,

2. Proceso de exploración, en donde "los participantes tienen la oportunidad de comprender dónde, cómo y por qué divergen sus valores e intereses, y dónde se necesita negociación para abordar aspiraciones legítimamente diferentes" (Mitchell, 2005, p. 9).

La experiencia neerlandesa sobre el tema de aguas nos orientará a identificar las metas y direcciones sobre lo que hay que hacer - know how - lo que es requerido especialmente por países ribereños que necesitan financiamiento y el conocimiento neerlandés sobre el manejo eficiente de los recursos hídricos. El proceso de exploración -en el que actualmente se encuentra el proyecto- tiene la capacidad y tiempo necesario para conjuntar intereses de distintos actores, además que pueden comprender el problema cabalmente al tener la fortaleza de "mantener la perspectiva del sistema, pero es selectiva y enfocada" (Mitchell, 2005, p. 9). Además su producto esperado -Instituto Tecnológico Aplicado del Agua- puede brindar "continuidad e institucionalidad a los esfuerzos que los distintos actores, en torno al recurso hídrico" realizan (Acuña, 2017). Para la implementación de este concepto se necesita de "un conjunto de tareas específicas para el gobierno, a saber, estimular, mediar, participar en servicios de intermediación, 
Pablo Carrasco

Proyecto "GIRAgua": síntesis de la cooperación internacional

crear el derecho, condiciones, hacer cumplir sus leyes y participar en la dirección" (Loorbach \& Rotmans, 2010, p. 2-3).

Con respecto a la Gobernanza del Agua, en términos prácticos, sus objetivos son:

1. Análisis basado en el riesgo (relacionado con el aumento de la escasez del agua causada por el cambio climático).

2. Optimización del uso del agua y oportunidades de reutilización en áreas urbanas, por el sector agrícola y la industria minera.

3. Disponibilidad de gestión de datos, modelos, redes de monitoreo, indicadores existentes, y medidas actuales.

4. Buen gobierno y participación de los interesados.

Además, este concepto implica no solo "la búsqueda de soluciones inteligentes e innovadoras a los problemas del agua, sino que también tiene pericia y experiencia en la generación de apoyo para ellas, la participación de los interesados y la organización de la gestión de esas soluciones" (Dutch Water Authorities, 2014). Estos principios fueron desarrollados en el $6^{\circ}$ Foro Mundial del Agua de Marsella del año 2012 en donde la discusiones se basaron no solamente en qué es lo que había que hacer, sino también "quién hace qué y por qué, a qué nivel de gobierno y el cómo" (OCDE, 2015, p. 1).

La Gobernanza del Agua a nivel internacional pretende encontrar soluciones a largo plazo que se caractericen por (OCDE, 2015, p. 3):

1. Efectividad. Contribución de la Gobernanza en definir metas y objetivos sostenibles y claros de las políticas del agua en todos los órdenes de gobierno, en la implementación de dichos objetivos de políticas, y en la consecución de las metas esperadas.

2. Eficiencia. Maximizar los beneficios de la gestión sostenible del agua y el bienestar, al menor costo para la sociedad.

3. Transparencia. Creación de confianza entre la población, y en garantizar la inclusión de los actores a través de la legitimidad democrática y equidad para la sociedad en general.

\section{Cooperación Internacional}

La cooperación internacional consiste en relaciones de países con distintos grados de expertise y conocimientos interesados en dialogar y resolver juntos problemas no solamente nacionales, sino que desde una perspectiva mundial. Si bien "en muchas ocasiones se ha convertido en una trampa que contribuye a perpetuar la miseria y la dependencia pues transforma a los países subdesarrollados que dependen de la ayuda en Estados pasivos" (Ayllón, 2007, p. 32), aún en la base de este sistema de cooperación "deberían encontrarse criterios como la equidad y la solidaridad internacional pero no es menos cierto que, con demasiada frecuencia, nos deparamos con el criterio del interés mutuo" (Ayllón, 2007, p. 36), que es parte sustancial de los apoyos internacionales de ciertos países a otros.

La iniciativa "GIRAgua" surge a partir de las redes de cooperación entre la Corporación Regional de Desarrollo Productivo y el Gobierno Regional de Atacama, que "impulsan una serie de estrategias y programas para profundizar en el uso de tecnología e innovación en esta materia, y se acaba de adjudicar un fondo proporcionado por Holanda que busca la gestión integrada de Atacama" (Acuña, 2017). Para 
esto es esencial la participación de múltiples actores, como también la expectativa de "una de las partes de que la actuación seguida por la otra parte, o las otras partes si la cooperación fuese multilateral, en orden a lograr sus propios objetivos, le ayuda a realizar sus intereses y valores" (Ayllón, 2007, p. 26).

Así, una mejor definición de cooperación internacional para este caso es que la cooperación es llevada a cabo por "Estados-nación u organizaciones de éstos, actores sub-nacionales u ONG's de un país, con otro/s de estos actores perteneciente/s a otro/s país/es, para alcanzar objetivos comunes en el plano internacional y/o en el nacional de uno o más actores" (Socas \& Hourcade, 2009, p. 21). Sobre esta base, y en relación a la participación de diferentes actores que pueden "interactuar en acciones de cooperación a nivel internacional, el término puede ser desambiguado" (Socas \& Hourcade, 2009, p. 21), sea en Cooperación Internacional Centralizada avocada en la acción principal de los Estados u organizaciones creadas por éstos para ejercer sus funciones; o Cooperación Internacional Descentralizada, enfocada en la acción de sujetos que escapan del control del Estado para llevar adelante los diálogos necesarios para crear redes de cooperación que superen el espacio de las fronteras nacionales soberanas.

En las Relaciones Internacionales como disciplina el conflicto por el poder y la sobrevivencia son parte de los ámbitos fundamentales de su análisis y de la mayor parte de sus lecturas y aproximaciones teóricas; la cooperación cumple un rol fundamental ya que permite crear relaciones de dependencia en algunos casos, o para éste en especial, se trata de "la existencia de un acuerdo (expreso o tácito) sobre los aspectos esenciales de las transacciones o de las actividades a realizar" (Ayllón, 2007, p. 26), creando una cooperación financiera ya que consiste en "la donación de fondos (no reembolsable) o el otorgamiento de créditos blandos (reembolsable), generalmente para la implementación de programas que favorezcan al desarrollo del beneficiario" (Socas \& Hourcade, 2009, p. 25).

En este sentido dentro de las Relaciones Internacionales, el proyecto GIRAgua comparte muchos rasgos con el concepto de Cooperación Internacional Descentralizada, que se lleva a cabo por "organismos subnacionales públicos u organizaciones privadas sin fines de lucro" (Socas \& Hourcade, 2009, p. 21), aún cuando los primeros lazos de esta cooperación fueron establecidos por los Estados. Además, otro concepto central es la de Cooperación Internacional para el Desarrollo, "si es considerada por su contenido, puede ser de carácter general o sectorial, orientada a la toma de decisiones conjuntas, al establecimiento de normas internacionales o de carácter operativo en la implementación de medidas o programas concretos de desarrollo" (Ayllón, 2007, p. 26).

Debido a la implementación de una agenda de desarrollo -Agenda 2030 del Sistema de Naciones Unidaspara promover la igualdad, el crecimiento económico, mejorar la capacidad tecnológica y fomentar la innovación de los países del "Tercer Mundo", se acuña un concepto de Cooperación Internacional para el Desarrollo más bien reciente, pero adecuado al presente contexto en donde ciertos países que adolecen de sus propios problemas y de la incapacidad de estos mismos Estados para abordar de manera inteligente estos desafíos. Así, se define la Cooperación Internacional para el Desarrollo y Descentralizada, ya que el proceso origina "redes de interacción entre los actores del sistema, cooperación y conflicto, y sus manifestaciones extremas, la integración” (Ayllón, 2007, p. 26), etc. En estas redes de cooperación se tratan diversos temas contemporáneos, que hacen difícil la mancomunidad de un mismo interés, o la presencia de un solo actor. Hoy en día, se hace más necesario una pluralidad de actores que antes no tenían los espacios para defender sus intereses en un tema crítico como es la gestión de los recursos 
hídricos ya que "la gestión del agua es una actividad determinada con objetivos múltiples y en parte conflictivos para mantener y mejorar el estado de los recursos hídricos” (Pahl-Wostl, 2007, p. 50).

Como una contribución al análisis y propuestas de solución al problema del uso sostenible de los recursos hídricos que busca trabajar e incidir en distintos ámbitos del quehacer nacional de manera de plantear el uso eficiente del agua y fomentar en Chile el desarrollo de una eficiencia hídrica en el mediano y largo plazo, se han desarrollado desde la Agencia Chilena de Cooperación al Desarrollo (AGCID) cinco Criterios Orientadores (CO) para el desarrollo:

1. Concentración de programas y proyectos en América Latina y el Caribe con estrategias diferenciadas.

2. Eficiencia mediante acciones flexibles y replicables.

3. Responsabilidad mutua y articulación de estrategias.

4. Gestión basada en la participación, el aprendizaje y el intercambio de conocimiento; y,

5. Fortalecimiento de las capacidades de la Agencia.

En esta perspectiva, el país donante y el país receptor de este sistema de cooperación y asistencias técnicas -y siendo un elemento importante a considerar en el futuro- se reúnen en una asociación indisociable. Uno de los elementos centrales de la Cooperación Internacional Centralizada es la condicionalidad de la ayuda prestada por un país hacia otro; es decir, "dar algo a cambio de que se cumplan determinados requisitos o condicionalidades” (Ayllón, 2007, p. 36). Esto considerando la perspectiva de que los países en menor grado de desarrollo deben prestar algún tipo de "servicio" a cambio o simplemente cumplir con ciertos requerimiento políticos o económicos a futuro para compensar la ayuda recibida, ya que "cada una de las transacciones se administra con el principal objetivo de promover el desarrollo y el bienestar económico de los países beneficiarios" (Ayllón, 2007, p. 37).

"GIRAgua" en definitiva ofrece excelentes oportunidades para la instalación de nuevos paradigmas que antes no eran considerados, como también aplicaciones innovadoras en donde las medidas y recomendaciones para promover el desarrollo de nuevas capacidades y proyectos debían ser implementadas gradualmente para enfrentar el cambio climático. Para conseguir tal objetivo, la "impermeabilización del clima debe ser impulsada por las oportunidades de innovaciones tecnológicas, institucionales y sociales, en lugar de simplemente por el temor a los efectos negativos del cambio climático" (Van Vierssen \& Kabat, 2005, p. 283).

\section{Experiencia internacional neerlandesa: Global Water Partnership (GWP).}

La presente sección presenta y caracteriza una institución de alcance global a través del cual es posible enfrentar los desafíos de un mundo que necesita mejorar el uso racional y eficiente de este recurso: la “Asociación Mundial del Agua” (Global Water Partnership, GWP por sus siglas en inglés).

Esta institución técnica especializada en el tema de aguas nace en 1996 como una organización intergubernamental con apoyo de la Agencia Sueca Internacional de Desarrollo (Swedish International Development Agency o SIDA por sus siglas en inglés), el Programa de Desarrollo de las Naciones Unidas (PNUD) y el Banco Mundial, con la misión de "apoyar a los países en el manejo sustentable de sus recursos hídricos" (Global Water Partnership International, 2010, p. 8), y bajo los principios de la Declaración de Dublín de 1992 sobre Gestión Integrada de los Recursos Hídricos. Tiene su sede en Estocolmo y también participan 8 países que figuran como "donantes" tanto para el mantenimiento 
financiero de la organización, como también para la consecución de sus objetivos, entre ellos, Argentina, Dinamarca, Chile, Hungría, Jordania, Paquistán, Países Bajos y Suecia, quienes nombran al Presidente del Comité Directivo. De su misión principal, se desprenden otros dos componentes que también dejaron su marca en la misma declaración de 1992:

1. Identificar las brechas y estimular a los socios a satisfacer necesidades críticas dentro de sus recursos humanos y financieros disponibles.

2. Apoyar la acción a nivel local, nacional, regional o de cuenca hidrográfica que siga los principios de la gestión sostenible de los recursos hídricos; y

3. Ayudar a unir las necesidades con los recursos disponibles (Global Water Partnership International, 2010, p. 8).

Esta organización promovía en sus orígenes el apoyo a los países para el manejo sustentable a través de los principios de la GIRH, que es definida aquí como "un proceso que promueve el desarrollo de la gestión coordinada del agua dulce, la tierra y los recursos relacionados con el fin de maximizar el bienestar económico y social resultante de manera equitativa son comprometer la sustentabilidad de los ecosistemas vitales" (Global Water Partnership International, 2010, p. 17). Por tanto, uno de sus principios generales en concordancia con la OCDE, era no tratar el agua "de manera aislada, y de hecho se deben considerar en relación con los problemas de la planificación de la tierra y el uso de la tierra” (Mitchell, 2005, p. 1336).

Existen dos periodos claramente definidos en la historia de esta organización: la primera de ellas, desde su creación en 1996 en donde su status legal sufrió una transformación, siendo la SIDA propietaria de su gobernanza, reemplazada por una nueva nomenclatura y una nueva forma de organización. Posteriormente, desde 2002 en adelante, la GWP se erige como una organización intergubernamental que proporciona redes de apoyo a países que lo necesiten a través de la ayuda de sus socios, agrupados en asociaciones de agua regionales, de países y de áreas geográficas específicas.

En esta nueva época, y a partir de esta misma transformación, entre 2004 y 2008 se desarrolló el denominado "Periodo Estratégico", donde se añadieron 1800 socios, formando una vasta red mundial interconectada de apoyo que a nivel regional, nacional y sub-nacional. Cada socio se compromete desde el 2008 en adelante a promover el concepto de GIRH como un medio para asegurar el manejo del agua, como también a:

1. Sensibilizar a los tomadores de decisiones (Decision Makers) sobre la importancia del agua y la necesidad de la GIRH

2. Compartir experiencias con otros socios

3. Proporcionar asesoramiento y servicios para ayudar a los socios de GWP donde y cuando sea apropiado; $y$

4. Formular prioridades, diseñar e implementar programas para resolver estas prioridades a través de enfoques de GIRH (Mitchell, 2005, p. 18).

Pero, ¿Cómo ha participado los Países Bajos en este organismo? Siendo uno de los países fundadores y donantes de GWP, junto con Suecia y el Reino Unido, financió el programa con US\$28.47 millones, que representa el 69\% de los ingresos totales de la organización desde el año 2008. Además, los Países Bajos han aportado a través del programa NWP (Netherlands Water Partnership), en alianza con el Banco Mundial, para posibilitar los planes de la GIRH a través de asistencia técnica y fondos fiduciarios para, por ejemplo, destinar recursos para apoyar el desarrollo sostenible en África como donante (US7.4 millones). 
En esta nueva época, el programa internacional GWP se proponía, a partir de la Cumbre Mundial sobre Desarrollo Sostenible de Johannesburgo del año 2002, profundizar el desarrollo y la gestión mejorada de los recursos hídricos, basados en una participación verdadera e inclusiva de las partes interesadas, proporcionando un vínculo directo con los ODM que no solo trataba con asiduidad la gestión integrada del agua, sino que también profundizaba en dotar al tema de una mayor relación con otras temáticas como pobreza, salud, educación y degradación ambiental.

\section{Netherlands Water Partnership}

"Netherlands Water Partnership" nace como un organismo técnico independiente creado por el sector público y privado holandés, apoyado financieramente por el Bank Netherlands Partnership Programme (BNPP). Su misión es estudiar y analizar las implicaciones de la crisis del agua y posibles estrategias para abordar las causas subyacentes y las tensiones que surgen de ella, ya que la misma institución funciona como un ente generador de conocimientos y experiencias que sirven para mejorar la gestión del agua alrededor del mundo.

Además, la NWP “proporciona comunicación para varios programas gubernamentales” (Schaap, 2016, p. 11), asistiendo a gobiernos, ministerios y ONGs para aportar las mejores prácticas sobre la Gobernanza del Agua, como también congregar a los usuarios del agua y actores para que dialoguen con el fin de mejorar gestiones y enfrentar problemas a través de la colaboración inter-institucional y la transparencia.

Koop \& Van Leeuwen, a través de los preceptos de la OCDE, definen este tipo especial de gobernanza como: “(...) la gama de sistemas políticos, sociales, económicos y administrativos que existen para desarrollar y gestionar los recursos hídricos y la prestación de servicios hídricos en diferentes niveles de la sociedad y para diferentes propósitos" (Koop \& Van Leeuwen, 2016, p. 11).

La red NWP como sistema de colaboración internacional sobre el tema del agua ofrece un paquete de medidas especializadas en el servicio exterior, que se distinguen en cuatro elementos:

1. Conocimiento. En donde proporcionan "información actualizada y relevante sobre mercados, temas y organizaciones del sector en el hogar y en el extranjero. La oficina de NWP también es el mostrador para las organizaciones extranjeras interesadas en el conocimiento y la experiencia neerlandesa dentro del sector del agua.

2. Redes. La NWP es una vasta red de expertise y conocimientos, que proporciona a los interesados "contactos adecuados, socios, emparejamiento, jóvenes talentos con ambiciones internacionales. Mantenemos relaciones con instituciones internacionales, gobiernos, embajadas y otras partes interesadas"

3. Visibilidad. Debido a que el programa ofrece "experiencia en la creación de una estrategia conjunta, mensaje y cartera, organizamos misiones comerciales, 'Pabellones de Holanda" en ferias comerciales, y ofrecemos vitrinas y campañas

4. Influencia. "Debido a nuestra red única, público-privada, tenemos una influencia en la agenda de la política de agua holandesa y el sector está conectado a la política. También hacemos esto para las agendas internacionales a través de nuestros socios internacionales" (Schaap, 2016, p. 74). 
$\mathrm{Al}$ igual que los reportes técnicos, de avances y de nuevos desafíos que publica todos los años la dirigencia técnica de la RijksWaterStaat, a través de la página del Ministerio de Infraestructura y Medio Ambiente neerlandés, Netherlands Water Partnership (NWP) también evidencia los potenciales focos de apoyo internacional para solucionar problemas en más de 20 países con presencia permanente de especialistas en el campo del desarrollo de programas de manejo eficiente de recursos hídricos. Esta asociación, de al menos 200 miembros, se caracteriza por aunar a diferentes actores en un mismo espacio en donde:

"Netherlands Water Partnership también ha mapeado los intereses y ambiciones del sector holandés para Chile a fin de proporcionar aportes sustantivos para una posible agenda de cooperación con Chile. Esto proporcionó el apoyo necesario para una visita posterior de Henk Ovink. Ivo Demmers participó en nombre del sector con la misión de Henk Ovink. Ahora Chile es un país de enfoque dentro de Partners for Water" (Schaap, 2016, p. 41).

La experiencia holandesa sobre gestión del agua a nivel mundial puede verse reflejada a través de la cooperación que ha realizado la misma institución en los cinco continentes y que se ven reflejada en su informe del año 2016: "exporta" conocimientos, estrategias y apoyos financieros a países tan diversos como Polonia e Italia en Europa; Bangladesh, India e Indonesia en Asia; en el contexto sudamericano los apoyos más permanentes y de más larga data se concentran en Colombia en donde "El objetivo es el conocimiento y la creación de capacidades para ofrecer soluciones para los problemas del agua, pero también para crear oportunidades para el sector holandés" (Schaap, 2016, p. 38).

Ahora bien, el programa no estuvo exento de procesos de transición que formalmente desarrollaron nuevos episodios de cambios importantes tanto en su conformación, como también en sus objetivos e incluso en el paradigma del trabajo en equipo. El Netherlands Water Partnership está dentro de la llamada "Composición de un escenario de Transición", siendo el termino escenario o arena explicativo del contexto de cambios incrementales.

En ese mismo sentido, "un proceso de arena es un proceso evolutivo con mutaciones continuas" (Loorbach \& Rotmans, 2010, p. 7), en donde desde el año 2016 hasta la actualidad, el mismo programa reformó sus canales de comunicación y de gestión ya que "el gobierno usó el Portal Internacional del Agua como un portavoz para información y documentos relacionados con la Ambición Internacional del Agua” (Schaap, 2016, p. 8).Ésta experiencia servirá prontamente para que se desarrollen nuevas iniciativas en el sector agua en Chile, ya que según las observaciones del Banco Mundial, se necesita proteger los derechos de agua de los grupos vulnerables ya que "la protección de los DAA de los grupos vulnerables es fundamental y se logra a través del reconocimiento por la propia Constitución de los usos consuetudinarios y a través de medidas adicionales para la protección especial de los DAA indígenas y de pequeños agricultores” (Banco Mundial, 2011, p. 8).

\section{Definición del sistema de subvenciones (WWSD)}

Existen algunas preguntas: ¿Cómo se expresa el programa de cooperación internacional que actualmente llevan los Países Bajos en el resto del mundo? ¿Quién lo financia? ¿Quién/es participan e intervienen en los proyectos?, y más precisamente, ¿Cómo se expresa la cooperación entre Chile y los Países Bajos? Para ser respondidas, primero debemos examinar el sistema de Waterveiligheid en Waterzekerheid Stedelijke Delta's -Administración de Riesgo de Inundación y Seguridad del Agua en Deltas Urbanos, en adelante WWSD-, sistema de proyectos a nivel internacional por los cuales los Estados, empresas e incluso las 
Pablo Carrasco

Proyecto "GIRAgua": síntesis de la cooperación internacional

alcaldías pueden pedir cofinanciamiento para proyectos de infraestructura, como así otras entidades, sean éstas estatales o del sector privado.

El actual programa holandés WWSD es un proceso de licitaciones basados en subsidios internacionales del programa Partners Voor Water -apoyado por el Estado holandés, el BNPP y otras instituciones como Deltares y Arcadis, así como los aportes de los demandantes del proyecto- para la gestión del riesgo de inundaciones y la gestión del agua tanto de Deltas urbanos como rurales, que actualmente se extiende desde el año 2016 hasta el 2021. Su misión institucional es reunir a los actores interesados para mejorar la posición internacional del sector del agua holandés y así contribuir al problema mundial del agua, que se centra específicamente en las consecuencias del cambio climático.

Pero las responsabilidades sobre amenazas de inundaciones o la disponibilidad de agua potable para consumo no solo conciernen a las Juntas y a los Consejos Generales y Ejecutivos. Los y las alcaldes -a través de los casi 400 Municipios que conforman al país- tienen a su cargo "los planes de zonificación de sus municipios y la concesión de permisos de construcción” (Slomp, 2012, 35).

Los subsidios de capitales provienen del Estado neerlandés por una parte y de los denominados Think Tanks por otra, como empresas o comités de expertos - para el caso específico de Chile, los subsidiarios son Arcadis y Deltares- que conforman dentro del ya revisado "Triángulo de Oro" de la gestión hídrica neerlandesa aquellas entidades económicas que trabajan estrechamente con el Ministerio de Infraestructura y Medio Ambiente, y con la agencia estatal del agua RijksWaterStaat.

Como parte de este plan de cooperación internacional, el WWSD, a través del programa NWP, tiene presencia en los cinco continentes y ha levantado proyectos en países tan disimiles uno de otro, desde Colombia hasta Singapur y China. En Chile, el proyecto ha pasado el proceso de viabilidad y de aprobación de informes técnicos, con lo que se pretende a futuro consolidar este esquema de subsidios internacionales en la región de Coquimbo y en el Valle del río Elqui.

Dependiendo del problema que debe enmendarse, la WWSD decide en conjunto con la entidad demandante los protocolos a seguir, cuanta cantidad de capital debe ser aportado por cada uno de las entidades, -sea del receptor del mismo consorcio, como de las entidades neerlandesas- en qué plazos debe estar dividido el proyecto y, en primer lugar, el grado de éxito y de satisfacción de las necesidades a largo plazo.

Para ello, es necesario completar dos fases: en primer lugar, construir un Proyecto de Viabilidad para calificar la gestión del riesgo de inundación y el esquema de suministro de agua para el Delta urbano, donde la entidad demandante debe ser capaz de resolver un problema definido en un contexto regional. En segundo lugar, luego de esta fase de protocolo de viabilidad se pasa al Proyecto Piloto, donde se examinan las características del proyecto: sus ventajas, riesgos y si es factible encontrar una posible solución.

Siguiendo con esta idea y como está planteado en el proyecto GIRAgua, los proyectos de viabilidad técnica "son un portal para proyectos pilotos o proyectos directos de introducción al mercado", determinando la fuerza o debilidad del mismo, en tanto que también traza futuras líneas de investigación 
y posibilita solucionar problemas, si bien en un principio a escala regional, a escala nacional a través de la innovación tecnológica y de la experimentación en base a la gestión integrada.

En concreto, en las fases preliminares del proyecto de cooperación internacional, la WWSD funciona como un laboratorio: un proceso de experimentación donde las entidades demandantes y los socios del consorcio deben mostrar si su solución tiene suficientes posibilidades para desarrollar el proyecto a largo plazo en el contexto regional. Aquí se recopilan datos, cifras, y estadísticas anteriores que son suministradas por las entidades demandantes o por alguna oficina especializada que tenga en sus manos ciertos datos que serán la base del proyecto en sí mismo.

\section{Proyecto “GIRAgua” en Chile}

Tras haber definido cómo se ha desarrollado en los Países Bajos el programa de cooperación internacional de la NWP -a través del plan Global Water Partnership- en materia de gestión integrada de los recursos hídricos para los países en vías de desarrollo que son afectados por inundaciones, sequías y por el mal manejo de sus recursos, a continuación de explica como Chile ha llevado a cabo ésta cooperación internacional: quienes son responsables del proyecto, cuáles son sus objetivos actuales y sus pretensiones a futuro.

Así, bajo este esquema de Gobernanza del Agua estrechamente vinculada a los dominios que son fundamentales para el desarrollo, incluyendo la salud, el medio ambiente, la agricultura, la energía, la planificación espacial, el desarrollo regiona; la necesidad del manejo de ríos o de cuencas, de proporcionar sistemas de información y logística actualizados, de incorporar nuevas tecnologías de aguas, manejo sustentable del riesgo sobre las costas, en las que además se agregan otras áreas de cooperación como parte de la agenda bilateral. Los objetivos principales declarados en la carta son:

1. Desarrollo de un proyecto piloto en la cuenca del río Elqui a partir de los hallazgos del estudio GIRAgua, incorporando las áreas de Sistemas de Información, Transferencia de Tecnología, Gestión Integrada de los Recursos Hídricos.

2. Soporte para el diseño y desarrollo del ITA (Instituto de Investigación Tecnológica Aplicada) basado en la experiencia de los Países Bajos; y,

3. Evaluación de políticas públicas para la reducción de derrames en el sector de agua potable y saneamiento (Ministerio de Obras Públicas de Chile, 2016), vinculado al ODS $\mathrm{N}^{\circ} 6$ de la ONU.

Dado que el proyecto GIRAgua pretende resolver los problemas de Chile a partir del uso eficiente de los recursos hídricos, los conceptos de Gestión Integrada de Recursos Hídricos -en adelante GIRH- y de Gobernanza del Agua son trascendentales para entender la profundidad y complejidad del proyecto. A pesar de lo reducido de la "Carta de Intenciones", es la primera vez que se aplicaría en Chile un proyecto de tales características en términos de las políticas públicas sobre el tema del agua. Su relación con el concepto de GIRH es fundamental, ya que en los Países Bajos, "el enfoque de sistema de agua involucró la identificación de sistemas de aguas y el 'equilibrio' de las demandas sociales y los potenciales de estos sistemas" (Mostert, 2006, p. 20).

La necesidad de este instituto es el resultado de la comprensión de que muchas "organizaciones en el país están conectados con los recursos hídricos y necesitan trabajar juntos y compartir datos y mejores prácticas". Por tanto, según lo establece el formato de subsidio del proyecto mismo, el solicitante -es decir, los 
Proyecto "GIRAgua": síntesis de la cooperación internacional

beneficiarios del proyecto que son el Gobierno Regional de la Región de Coquimbo- ha considerado como objetivos prioritarios:

1. Análisis basado en el riesgo (relacionado con el aumento de la escasez del agua causada por el cambio climático

2. Optimización del uso del agua y oportunidades de reutilización en áreas urbanas, por el sector agrícola y la industria minera

3. Disponibilidad de gestión de datos, modelos, redes de monitoreo, indicadores existentes, y medidas actuales; $y$

4. Buen gobierno y participación de los interesados.

Pero ¿Cómo se hace efectivo el consorcio? ¿Cuáles son las organizaciones que apoyan el subsidio del proyecto? Dentro de su desglose presupuestario, se detallan 4 agentes o "socios del consorcio", y entre ellos, una secretaria. En el siguiente cuadro se especifican los socios, y su participación en el proyecto.

Figura 1. Consorcios participantes proyecto "GIRAgua".

\begin{tabular}{|c|c|c|c|c|}
\hline $\begin{array}{l}\text { Nombre } \\
\text { socio }\end{array}$ & Actividades principales & $\begin{array}{l}\text { Papel dentro del } \\
\text { proyecto }\end{array}$ & $\begin{array}{l}\text { Ambición en el país de } \\
\text { destino }\end{array}$ & $\begin{array}{l}\text { Experiencia en el país de } \\
\text { destino }\end{array}$ \\
\hline $\begin{array}{l}\text { Deltares } \\
\text { (Secretario } \\
\text { y socio del } \\
\text { consorcio). }\end{array}$ & $\begin{array}{l}\text { Riesgo de inundación, } \\
\text { planificación adaptativa del } \\
\text { delta, infraestructura }\end{array}$ & $\begin{array}{l}\text { Líder del proyecto, } \\
\text { análisis del sistema } \\
\text { de agua }\end{array}$ & $\begin{array}{l}\text { Aprovechar redes y } \\
\text { conocimiento para } \\
\text { impulsar inversiones } \\
\text { con posibilidades de } \\
\text { enfoques integrados } \\
\text { del agua. }\end{array}$ & $\begin{array}{l}\text { Experiencia en proyecto } \\
\text { relacionados con la } \\
\text { adaptación al clima, la } \\
\text { mitigación de la erosión } \\
\text { costera, escasez de agua e } \\
\text { inundaciones }\end{array}$ \\
\hline $\begin{array}{l}\text { LeAF } \\
\text { (Socio del } \\
\text { Consorcio). }\end{array}$ & $\begin{array}{l}\text { Investigación y consultoría } \\
\text { sobre tratamiento y } \\
\text { purificación de } \\
\text { residuales y y } \\
\text { orgánicos sólidos }\end{array}$ & $\begin{array}{l}\text { Tecnologías de } \\
\text { tratamiento de aguas } \\
\text { residuales }\end{array}$ & $\begin{array}{l}\text { Adquirir experiencia } \\
\text { en Chile, crear } \\
\text { oportunidades para } \\
\text { proyectos futuros }\end{array}$ & $\begin{array}{l}\text { Escasa: escritura conjunta } \\
\text { de propuestas con la } \\
\text { Universidad Adolfo Ibáñez } \\
\text { en el futuro }\end{array}$ \\
\hline $\begin{array}{l}\text { Fundación } \\
\text { IHE Delft- } \\
\text { UNESCO. } \\
\text { (Socio del } \\
\text { consorcio). }\end{array}$ & $\begin{array}{l}\text { Educación hídrica } \\
\text { multidisciplinaria a nivel de } \\
\text { postgrado, capacidad de } \\
\text { desarrollo e investigación } \\
\text { orientada a problemas de } \\
\text { América Latina }\end{array}$ & $\begin{array}{l}\text { Dirige el paquete de } \\
\text { trabajo sobre análisis } \\
\text { de interesados y } \\
\text { agua; análisis de } \\
\text { conflictos de agua }\end{array}$ & $\begin{array}{l}\text { Ampliar colaboración } \\
\text { con Chile en las áreas } \\
\text { de educación graduada } \\
\text { en agua (maestría, } \\
\text { doctorado), } \\
\text { intercambio de } \\
\text { personal universitario }\end{array}$ & $\begin{array}{l}\text { Diversos proyectos de } \\
\text { desarrollo de capacidades } \\
\text { de estudiantes de maestría y } \\
\text { doctorado de Chile; amplia } \\
\text { experiencia en el desarrollo } \\
\text { de capacidades (aplicadas) } \\
\text { en proyectos de } \\
\text { investigación en América } \\
\text { Latina }\end{array}$ \\
\hline $\begin{array}{l}\text { Arcadis } \\
\text { Chile. } \\
\text { (Socio del } \\
\text { consorcio). }\end{array}$ & $\begin{array}{l}\text { Empresa líder en Chile en } \\
\text { áreas de minería, } \\
\text { infraestructura, energía, } \\
\text { medio ambiente y el agua }\end{array}$ & $\begin{array}{l}\text { Arcadis estará a } \\
\text { cargo del diseño del } \\
\text { proyecto piloto y } \\
\text { escenarios futuros. }\end{array}$ & $\begin{array}{l}\text { Aplicar la metodología } \\
\text { desarrollada a partir de } \\
\text { este proyecto a otras } \\
\text { regiones en Chile }\end{array}$ & $\begin{array}{l}35 \text { años de experiencia en } \\
\text { Chile. La división de agua } \\
\text { tiene mucha experiencia } \\
\text { trabajando en proyectos } \\
\text { tanto para instituciones } \\
\text { públicas como privadas }\end{array}$ \\
\hline
\end{tabular}


Fuente: creación propia a partir de datos de programa de subsidios WWSD, "GIRAgua” Chile. 2016.

Según los propósitos del proyecto, GIRAgua ha identificado diferentes soluciones sostenibles y a corto y largo plazo, entre las que se encuentran:

1. Medidas de adaptación que aumente la recarga de acuíferos o napas subterráneas.

2. Mayor eficiencia del uso del agua por parte de la industria agrícola (a pequeña y gran escala), y la minería; $y$,

3. Tecnologías para la minimización del uso del agua

Este enfoque IRBM dentro del estudio de factibilidad, pretende trabajar con la cuenca del río Elqui estableciendo diversos parámetros de análisis y de mitigación de los efectos adversos de las sequías y la escasez de aguas.

\section{Conclusiones y propuestas.}

Como se ha expuesto en esta investigación, se ha expuesto la crítica situación del manejo del agua en el país, como también las principales necesidades del país para mejorar los estándares del uso del agua y que conceptos tales como "Gobernanza del Agua” y "Gestión Integrada de los Recursos Hídricos” sean parte de una nueva política pública hídrica para el país.

Para ello, es necesaria la implementación del manejo integrado de los recursos hídricos. Primero, debido a la presión intensiva sobre los recursos hídricos, se hace imperioso políticas públicas coherentes, transparentes, efectivas y eficientes, como están planteados en los principios de la Gobernanza del Agua y cómo se desarrollan los mismos en la institucionalidad neerlandesa. En segundo lugar, para alcanzar un manejo integrado y eficiente, es necesario contar con una institucionalidad capaz de resolver las necesidades y retos de un problema tan multi-dimensional como el agua, ya sea para evitar los periodos de escasez como aquellos episodios no previstos, como inundaciones y maremotos. En tercer lugar, es tarea de todos los actores de la sociedad como ministerios, instituciones como secretarías y departamentos del gobierno, sector público y privados, y usuarios del agua crear contextos de diálogos y cooperación entre los distintos actores: crear una cultura hídrica que haga posible consecuencias beneficiosas para el país y mejorar los estándares del manejo de agua en base a una economía sostenible.

Se trata no solo de enfrentar los problemas derivados de la provisión de agua para la población o evitar los efectos de la contaminación de las aguas superficiales y/o de las napas subterráneas como queda demostrado en las Cuentas Presidenciales: los nuevos requerimientos para una gestión integrada del agua involucran una nueva relación con el agua, el uso de la tierra, e introducir nuevos paradigmas como "Seguridad Hídrica" y "Gobernanza del Agua".

Como propuesta, delinear un plan a partir de la experiencia holandesa, urge la instalación y fomento del Instituto de Tecnología Aplicada (ITA) en cada región o sistemas de Deltas para generar conocimiento e información sobre la gestión de los ríos en el país, y por tanto es urgente que la cooperación al desarrollo de nuevas políticas públicas hídricas institucionales (e incluso iniciativas propias de las Juntas de Agua y de la sociedad civil) que en Chile y los Países Bajos se incorporarían con la firma del proyecto "GIRAgua", deben alinearse con la "Declaración de Dublín en consonancia con la "Seguridad Hídrica" y sus principios, siendo éste el inicio de la cooperación que Chile debe fortalecer en el futuro. 
Para terminar, en conclusión es necesario incorporar más a la sociedad civil en las discusiones y debates en el diseño de las políticas públicas en torno al tema del agua y del medio ambiente ya que se hace imprescindible que las Juntas de Vecinos, asociaciones de la sociedad civil como ONG y Fundaciones, participen activamente y que la institucionalidad considere relevante las proposiciones devenidas de la misma sociedad civil, aunque en Chile se justifique la dificultad de la adopción de este proyecto, ya que el director nacional de la DGA indica que "es difícil lograr que todos trabajen coordinados porque hay muchos intereses, pero sí es importante que haya un conocimiento común de la disponibilidad del recurso, de la fuente a la cual se puede acceder, y que eso sirva de común acuerdo para todos los usuarios" (Paleo, 2019).

\section{Referencias}

Acuña, A. (2017) "Región de Coquimbo se adjudica proyecto que busca uso eficiente de los recursos hídricos. Acceso el 6/05/2019. EN: http://www.elobservatodo.cl/noticia/sociedad/region-decoquimbo-se-adjudica-proyecto-que-busca-uso-eficiente-de-los-recursos-hid

Ayllón B. (2007). La cooperación internacional para el desarrollo: fundamentos y justificaciones en la perspectiva de la Teoría de las Relaciones Internacionales. Revista Carta Internacional.

Banco Mundial. (2011). Diagnostico gestión de recursos hídricos en Chile.

Dutch Water Authorities (2014). Informe Oficial Final Dutch Water Authorities. La Haya. P. 3. EN: https://www.dutchwaterauthorities.com/wp-content/uploads/2015/09/Leaflet-Dutch-WaterAuthorities-Spanish.pdf

Fajardo, M. \& De Britto, G. (2017) "Experto holandés advierte que Chile no debe esperar a que haya un desastre para enfrentar la crisis del agua". El Mostrador. Acceso el 10/03/2019. EN: http://www.elmostrador.cl/cultura/2017/01/12/experto-holandes-advierte-que-chile-nodebe-esperar-a-que-haya-un-desastre-para-enfrentar-la-crisis-del-agua/

Global Water Partnership International (2010). Global Program Review. Volume 4, Issue

Informe Técnico Proyecto GIRAgua. 2016

Koop, S. \& Van Leeuwen, K. (2016). The challenges of water, waste and climate change in cities. Environment Development and Sustainability Review. EN: https://www.researchgate.net/publication/292669720 The challenges of water waste and climate change in cities

Loorbach, D. \& Rotmans, J. (2010). The practice of transition management: Examples and lessons from four distinct cases. Future Review. Volume 42, Issue 3. EN: https://www.researchgate.net/publication/222518552_The practice of transition_manageme nt Examples and lessons from four distinct cases

Ministerio de Obras Públicas/Dirección General de Aguas. (2014). Atlas del Agua. Capítulo I.

Ministerio de Obras Públicas de Chile y el Ministerio de Infraestructura y Medio Ambiente de Holanda. (2016). Carta de Intenciones.

Mitchell, B. (2005). Integrated Water Resource Management, institutional arrangements, and land-use planning. Environment and Planning Review. Vol. 37.

Mostert, E. (2006). Integrated Water Resources Management in the Netherlands: How concepts function. RBA Center: Delft University of Technology. Netherlands. EN: https://onlinelibrary.wiley.com/doi/abs/10.1111/j.1936-704X.2006.mp135001003.x

OCDE. (2015). Principios de Gobernanza del agua de la OCDE. Consejo Ministerial OCDE/Junta 
Directiva de Gobernanza Pública y Desarrollo Territorial

Pahl-Wostl, C. (2007). Transitions towards adaptive management of water facing Climate and Global Change. Water Resource Manage Review. University of Osnabruck. Germany. 21: 49-62.

Paleo, D. (2019). DGA reconoce que proyecto GIRAgua se podría replicar en el resto del país. Diario El Día. Acceso el 8/4/2019. EN: http://www.diarioeldia.cl/economia/agua/dga-reconoce-queproyecto-giragua-se-podria-replicar-en-resto-pais

Schaap, S. (2016). Meer impact in het buitenland. Netherlands Water Partnership International Technical Inform.

Socas, N. \& Hourcade, O. (2009). "La cooperación Internacional” EN: CHIANI, Ana María et. Al. Coordinado por SCARTASCINI, J. La Cooperación Internacional: herramienta clave para el desarrollo de nuestra región. $1^{\text {a }}$ Edición. Buenos Aires: Konrad Adenauer Stiftung. P. 21. EN: https://www.kas.de/c/document library/get file?uuid=c920df51-f9ec-b210-e38d2bbe7024dd3c\&groupId $=287460$

Slomp, R. (2012). Riesgo de inundación y Gestión del agua en los Países Bajos. Ministry of Infrastructure and the Environment. EN: file:///C:/Users/Usuario/Downloads/overstromingsrisico en water beheer in nederland d e stand van zaken in 2012 rijkswaterstaat.pdf

Van Vierssen W. \& Kabat, P. (2005). Climate proofing the Netherlands. Nature Review. No 438. EN: https://www.nature.com/articles/438283a

Zwarteveenn, M. \& Boelens, R. (2014). Defining, researching and struggling for water justice: some conceptual building blocks for research and action. Water International Review. $\mathrm{N}^{\circ}$ 39, Vol. 2. EN:

http://www.cedla.uva.nl/20_research/pdf/Boelens/Zwarteveen\&Boelens $\% 20$ WaterJustice $\% 20$ WatInt ernat2014.pdf 